\title{
Safety and efficacy of incobotulinumtoxinA as a potential treatment for poststroke spasticity
}

REVIEW

This article was published in the following Dove Press journal:

Neuropsychiatric Disease and Treatment

27 January 2016

Number of times this article has been viewed

\begin{abstract}
Andrea Santamato
Physical Medicine and Rehabilitation Section, "OORR Hospital," University of Foggia, Foggia, Italy
\end{abstract}

Video abstract

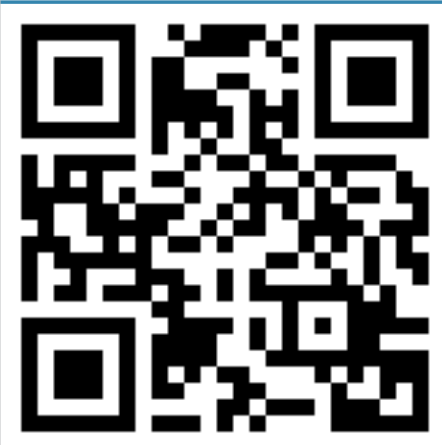

Point your SmartPhone at the code above. If you have a QR code reader the video abstract will appear. Or use:

http://youtu.be/6TSvklufg8w
Correspondence: Andrea Santamato Physical Medicine and Rehabilitation Section, "OORR Hospital," University of Foggia, Viale Pinto, 7I I 00 Foggia, Italy Tel $+3988 I 736258$

Fax +39881732564

Email andrea.santamato@unifg.it
Abstract: Spasticity is a common disabling symptom for several neurological conditions. Botulinum toxin type A injection represents the gold standard treatment for focal spasticity after stroke showing efficacy, reversibility, and low prevalence of complications. In recent years, incobotulinumtoxinA, a new Botulinum toxin type A free of complexing proteins, has been used for treating several movement disorders with safety and efficacy. IncobotulinumtoxinA is currently approved for treating spasticity of the upper limb in stroke survivors, even if several studies described the use also in lower limb muscles. In the present review article, we examine the safety and effectiveness of incobotulinumtoxinA for the treatment of spasticity after stroke.

Keywords: Hypertone, botulinum toxin, incobotulinumtoxinA, onabotulinumtoxinA, abobotulinumtoxinA

\section{Introduction}

Spasticity with muscle paresis and loss of dexterity represents one of the most common and discomforting complications affecting stroke survivors. It can have a disabling effect on stroke patients through pain and reduced mobility, affecting quality of life, and can be highly detrimental to daily functioning. Previous studies, based on the estimates of health care professionals, suggested that the prevalence of poststroke spasticity was $\sim 60 \%$, even if this value can be lower than the real value considering the difficulties in measuring spasticity routinely in rehabilitative settings. ${ }^{1}$

In a recent study, conducted in a clinical setting, 39\% of patients with first-ever stroke were spastic after 12 months. ${ }^{2}$ Lundström et al reported that an estimated prevalence of spasticity 1 year after the first-ever stroke was $17 \%$ and that it was more prevalent in the upper limb than in the lower limb. ${ }^{3}$

In another study, spasticity was present in only $19 \%$ of the 95 subjects investigated 3 months after stroke. ${ }^{4}$ The same group of authors reported that 13 subjects out of 63 displayed spasticity after 18 months of stroke. ${ }^{5}$

There is no consensus concerning the number of patients developing spasticity. The discrepancies about the prevalence of spasticity onset after stroke might be related to various study settings and samples as well as the difficulty to measure and to identify its early development, discriminating between spastic-dystonia, muscle contracture, increase of stiffness, and other biomechanical factors. It is known that spastic hypertone can be responsible for motor impairments and activity limitations as well as for forced limb posture and pain at rest and during passive movements. The degree of spasticity may change according to the position of the patients, the task being performed, and the presence of aggravating factors such as pressure ulcers, skin infections, or urinary tract infections. Therefore, considering the variability of clinical features of stroke survivors, 
the assessment of spasticity is difficult as well as the need for treatment. In fact, for example, it has also been suggested that for stroke patients, the overactivity of leg extensor muscles enables them to support their body, standing position, and stance phase of gait cycle but interferes with knee flexion during the swing phase, so in this case, a botulinum toxin (BoNT) injection into rectus femoris or vastus intermedius muscles can be useful to reduce this impairment. ${ }^{6}$

Spasticity is also divided into generalized and focal when few muscles are involved. This type of classification can influence the choice of treatment considering not only the therapy but also the aim to improve limb posture and body image, to apply splinting, to consent hygiene, to increase passive articular range of motion, to walk and stand, to decrease pain and discomfort, to reduce the burden of care, or to prevent contracture. The purpose of this review article is to evaluate the effectiveness of the employment of incobotulinumtoxinA, a recent marked formulation of botulinum toxin type A (BoNT-A), to reduce spasticity in stroke survivors through an analysis of published clinical studies.

\section{Management of poststroke spasticity}

The treatment of spasticity is strictly related to the reason by which the physicians prefer to obtain a muscle hypertone reduction for the patients. Several types of treatments can be used, even if the choice depends on the characteristics of the muscle overactivity, if generalized (oral drugs, intrathecal baclofen, intrathecal phenol, neurosurgery) or focal (BoNT injection, phenol nerve blocks, orthopedic surgery, physical therapies). In all cases, all pharmacological interventions are adjunctive to a program of rehabilitative treatment. ${ }^{7,8}$ Therefore, a variety of options are available, and clinical experience has shown that a multimodal approach is more effective than single therapy.

Oral antispastic drugs represent a therapeutical possibility to reduce muscle hypertone in poststroke survivors. Although spasticity after stroke is considered a focal muscle condition that requires a local therapy, many clinicians prefer the use of systemic drugs. There are several aspects that must be considered using oral drugs, ie, side effects, no evidence-based guidelines for the choice, titration rates, and withdrawal of these drugs. Among the side effects, one of the most important is the muscle weakness, especially at high dosages. Many physicians consider that it could be related to unmasking of underlying upper motor neuron syndrome rather than from a direct drug effect. Moreover, the onset of dizziness, sedation, and hypotension could be responsible for the withdrawal. Often spasticity is not well treated, and drugs are too quickly interrupted if not tolerated. A combination of two drugs could be used to increase the effect of a single agent or if the patient can tolerate only low doses.

Among oral drugs, baclofen, benzodiazepines, gabapentin, and pregabalin act on the system of gamma-aminobutyric acid (GABA). Baclofen is a GABA-B receptor agonist: it reduces calcium influx and suppresses the release of excitatory neurotransmitters, including glutamate and aspartate. It downregulates the activity of 1a sensory afferents, spinal interneurones, and motor neurons. ${ }^{9}$ The possibility of using baclofen through intrathecal administration reduces the adverse effects related to the oral administration. In fact, oral baclofen has a very low bioavailability to GABAergic neurones in the spinal cord, whereas intrathecally, a relatively small dose of drug is given directly into the spinal cord at a high concentration. This employment consents to reduce side effects with a good therapeutical effect of muscle relaxation. However, this type of administration is used less to reduce spasticity after stroke, which often requires a selective treatment. ${ }^{10}$ Benzodiazepines act on GABA-A receptors. They have similar efficacy to other antispasticity drugs but with more side effects. ${ }^{11}$ Gabapentin and pregabalin showed their effectiveness especially in the case of spasticity-related pain. It requires a great compliance by the patient considering the possibility of also taking 3,600 mg/daily of these drugs. ${ }^{12}$ Tizanidine is an $\alpha 2$ adrenergic receptor agonist that enhances noradrenergic activity in the spinal cord and brain and inhibits excitatory spinal interneurones. ${ }^{13}$ Dantrolene acts directly on the muscle blocking calcium release from the sarcoplasmic reticulum and reduces the excitation-contraction coupling of the skeletal muscle. ${ }^{14}$ It is less sedative than other oral drugs, but it is hepatotoxic. In the last few years, cannabinoids have been used to treat spasticity and pain related to multiple sclerosis, acting on receptors located in the dorsal spinal cord, basal ganglia, hippocampus, and cerebellum. Sedation and psychotropic side effects are predominant with the use of tetrahydrocannabinol, an agonist of cannabinoid 1 and 2 receptors, whereas are less frequent with cannabidiol. There is a lack of data about its effectiveness, as occurs for other pharmacological therapies, and a previous study found no significant reduction in the objective measures of spasticity. ${ }^{15}$

Among physical therapies to reduce spasticity, neuromuscular electrical stimulation represents the most used, even if the difficulty to employ it depends on the presence of a severe spasticity, the presence of biomechanical muscle alteration (tendon retraction, fibrosis), the lack of specific protocols, and the data about amplitude and frequency. ${ }^{16}$ 
The effectiveness of muscle stretching for spasticity treatment has been recognized recently, but the available evidence on its clinical benefit is overall inconclusive. ${ }^{17}$ Dorsal rhizotomy is used to reduce generalized spastic hypertonia, as occurs in cerebral palsy or in particular cases of stroke. This treatment reduces the sensory input into spinal-motorneuron pools, reducing their excitability. ${ }^{18,19}$ Orthopedic surgery (tendon lengthening, soft tissue release, and osteotomies) may be considered in patients with upper and lower limb spasticity after stroke, particularly for equinovarus foot deformity, improving kinetic and kinematic gait parameters. ${ }^{20}$ However, further validation of surgical correction of spastic foot following stroke is desirable with higher level of study designs and validated assessment tools. A selective treatment is the phenol nerve blockade directly into the peripheral nerves. This treatment causes destruction of neural tissue by protein coagulation even if nerve sprouting may lead to recurrence of spasticity. Thigh adductors and plantar flexor muscles are more frequently treated. It should be done only under the guidance of an ultrasound scan or nerve stimulator. ${ }^{21}$

The gold standard therapy for focal spasticity after stroke is BoNT-A, derived from the anaerobic bacterium Clostridium botulinum. The toxin molecule is composed of a light chain $(50 \mathrm{kD})$ and heavy chain $(100 \mathrm{kD})$, linked by a disulfide bond. The heavy chain is responsible for neuron internalization, and the light chain cleaves specific target proteins involved in the docking and fusion of acetylcholine-containing vesicles to the internal portion of the cell membrane. These target proteins are collectively referred to as the SNARE complex (soluble $N$-ethylmaleimide-sensitive fusion protein). The light chain of BoNT-A cleaves a protein termed SNAP-25, blocking the fusion of acetylcholine-containing vesicles to the cell membrane and acetylcholine release. ${ }^{22}$ The effect is reversed by nerve sprouting and reinnervation, which develops over a few months. If required, further injections should be planned after 3-4 months. An accurate injection technique is required to better identify target muscles, to obtain the identified goal, and to reduce the spread of toxin around near tissues. ${ }^{23}$ Management of stroke patients submitted to BoNT-A therapy is necessary to increase the relaxant effect within the treated muscle. ${ }^{24}$ Adverse events of BoNT include respiratory tract infections, muscle weakness, urinary incontinence, fever, pain, and transient dysphagia.

\section{BoNT-A for the treatment of poststroke spasticity}

Since 1989, BoNT-A has been shown to be effective in reducing spasticity after stroke with reversibility and low prevalence of complications, obtaining the approval of US Food and Drug Administration and European regulatory agencies for this indication. ${ }^{25}$ In the following years, many studies have demonstrated its safety and effectiveness, ${ }^{26,27}$ although controversy also exists about the improvement in motor function relative to the improvement in spasticity. Several international consensus statements on dosages, injection techniques, patient selection, and outcome measures have been proposed, ${ }^{28-30}$ even if injection sites and dosage are often identified by the injector's decision-making without scientific basis and specialized training. There is a general agreement on the need for physiotherapy, but a general consensus on timing (eg, whether to start immediately or days or weeks after BoNT-A injections), duration (eg, how long a rehabilitation program should last), type of rehabilitation procedures (eg, multimodal or single procedure or both), as well as the cost-effectiveness of such treatments has not yet been reached.

Current guidelines suggest the employment of a dose up to 600 units $(\mathrm{U})$ of onabotulinumtoxinA and incobotulinumtoxinA or up to $1,500 \mathrm{U}$ of abobotulinumtoxinA per injection session to treat spasticity after stroke. ${ }^{28}$ However, in recent years, higher doses were used, especially in the case of upper and lower limb severe spasticity, to improve limb posture, to apply splinting, to consent hygiene, and to increase passive articular range of motion (ROM). ${ }^{31,32}$ To date, four formulations of BoNT-A are commercially available and used in clinical practice: onabotulinumtoxinA (Botox ${ }^{\circledR}$; Allergan, Inc., Irvine, CA, USA), abobotulinumtoxinA (Dysport ${ }^{\mathbb{R}}$; Ipsen, Paris, France), incobotulinumtoxinA (Xeomin ${ }^{\circledR}$; Merz Pharma GmbH, Berlin, Germany), and a Chinese toxin (Prosigne ${ }^{\circledR}$; Lanzhou Institute of Biological Products, Lanzhou, People's Republic of China). The preparations are manufactured by different processes and have various formulations and potencies, which are determined by diverse biological assays based on their clinical use. ${ }^{33}$

As largely described in several studies, ${ }^{34,35}$ between onabotulinumtoxinA and incobotulinumtoxinA, there is no difference in potency. In contrast, the conversion ratio between abobotulinumtoxinA and incobotulinumtoxinA or onabotulinumtoxinA is not yet clear. Recently, it has been shown that $100 \mathrm{U}$ of onabotulinumtoxinA or incobotulinumtoxin A is bioequivalent to $300 \mathrm{U}$ of abobotulinumtoxin . $^{33,36}$ Another interesting consideration about the commercially available BoNT-A products is their protein structure. The active component of the available products is BoNT in the onabotulinumtoxinA and abobotulinumtoxinA formulations, the neurotoxin is associated with a larger protein complex 
containing accessory proteins, whereas incobotulinumtoxinA formulation presents a neurotoxin purified, free from complexing proteins, and thus has a high specific biological activity. ${ }^{37}$ It has been demonstrated that, under physiological conditions, complexing proteins are not associated with the neurotoxin, and so are not required for the pharmacological activity of the neurotoxin and do not affect the stability of the products or the diffusion after injection. ${ }^{38-40}$ The advantage of the absence of complexing proteins could be related to low risk of immunogenicity, but this topic is not yet established. A recently published case report showed the effect of incobotulinumtoxinA to reduce spasticity in a stroke survivor who developed BoNT-A antibodies after several years of treatment with onabotulinumtoxin $\mathrm{A}^{41}$

In the present review article, we examined the safety and effectiveness of incobotulinumtoxinA for the treatment of spasticity after stroke (Table 1). We reviewed English clinical reports from the international literature published from January 2009 to October 2015, including randomized placebocontrolled, double-blind, and open-label trials, and existing meta-analyses that provided a description of the employment of incobotulinumtoxinA for the treatment of spasticity after stroke. We chose this starting date for the electronic searches because between 2009 and 2010, incobotulinumtoxinA was approved in Europe for the treatment of upper limb poststroke spasticity. ${ }^{42}$ Searches of US National Library of Medicine databases were performed and were included if 1 ) the sample size included four or more subjects; 2) the intervention applied was incobotulinumtoxinA; 3) spasticity was assessed pre- and posttreatment using specific outcome measures; and 4) safety was described by the clinicians. We did not include congress abstracts/posters, articles that were not peer reviewed, articles not written in English, and case reports. This review was based upon searches of the following terms in the US National Library of Medicine databases: "incobotulinumtoxinA" or "NT201" or "Xeomin" ${ }^{\circledR}$ " combined with "spasticity" or "poststroke spasticity." The references of each study selected were screened to identify studies that were not included on electronic search. Key textbooks were also searched in addition to the electronic database search. From 146 articles identified with multiple electronic searches, we screened titles and abstracts of the citations downloaded from the searches identifying 46 potential relevant articles chosen for a closer review. Excluding other 32 articles not meeting the inclusion criteria, we obtained full copies of the 14 potentially suitable reports for further assessment. After inclusion of two articles of interest from the reference lists of the selected articles and exclusion of an other four articles,
12 studies met the study eligibility criteria and were finally included in the overall review (Table 1). ${ }^{23,31,43-52}$

\section{IncobotulinumtoxinA for the treatment of poststroke spasticity}

According to the European product label, incobotulinumtoxinA is currently approved for treating upper limb spasticity in stroke survivors. In 2010, the European Medicines Agency declared that incobotulinumtoxinA was effective to reduce upper limb spasticity after stroke with the use of $400 \mathrm{U}$ of neurotoxin. ${ }^{42}$ A great contribution to the research about the use of incobotulinumtoxinA for the treatment of spasticity after stroke was given by a randomized, double-blind, placebo-controlled, Phase III study carried out at 23 sites in three European countries on 148 stroke survivors with upper limb spasticity. ${ }^{43}$ The patients have been submitted to a single treatment session with incobotulinumtoxinA (maximum dose $400 \mathrm{U}$ ) or placebo administrated into fingers and wrist flexors, elbow flexors, forearm pronators, and thumb flexor muscle and followed for $\leq 20$ weeks. One month after the BoNT-A injections, a significantly higher proportion of patients treated in wrist flexors with neurotoxin were responders (improvement of $\geq 1$ point in the Ashworth Scale [AS] score), as observed in comparison to placebo $(P<0.001)$ as well as for all treated flexor muscle groups $(P \leq 0.009)$. Statistically significant results in favor of incobotulinumtoxinA were observed at all postinjection visits until week 12 in the principal therapeutic target for Disability Assessment Scale (DAS) $(P \leq 0.005)$ in the global assessment of efficacy $(P<0.001)$ and in some tasks of the Caregiver Burden Scale $(P<0.05)$. Approximately 20 subjects in each group experienced at least one of the following adverse event: feeling hot, headache, dysesthesia, hypoesthesia, dysphagia, injection site pain, and injection site hematoma. ${ }^{43}$

In an open-label extension period of this trial with up to a duration of 69 weeks, most patients (145/148) received a maximum of five additional sets of incobotulinumtoxinA injections with $\geq 12$ week intervals. ${ }^{44}$ One hundred and twenty patients completed the extension period, and clinical effects of the therapy confirmed previous results on spasticity reduction for wrist, elbow, finger, thumb flexors, and the forearm pronators with a significant improvement after 4 weeks (AS response rate: $\leq 80.6 \%, P<0.0001$ ). After 4 weeks of follow-up, DAS score showed a significant improvement $(P<0.05)$, and the majority of investigators, patients, and caregivers rated incobotulinumtoxinA efficacy as very good or good (56\%-84\%). Investigators rated the tolerability of incobotulinumtoxinA as "good/very good" for $\geq 90 \%$ of 


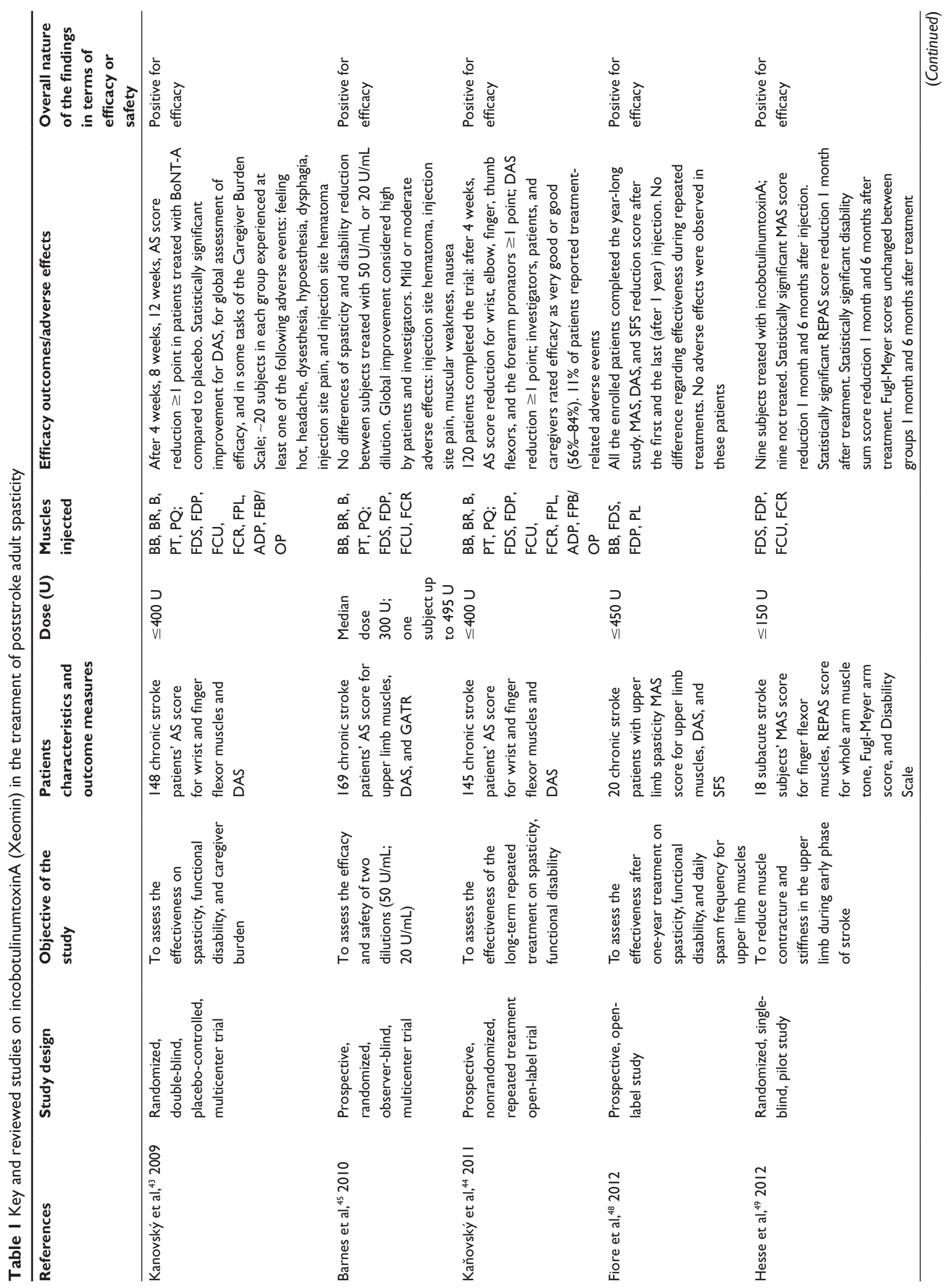




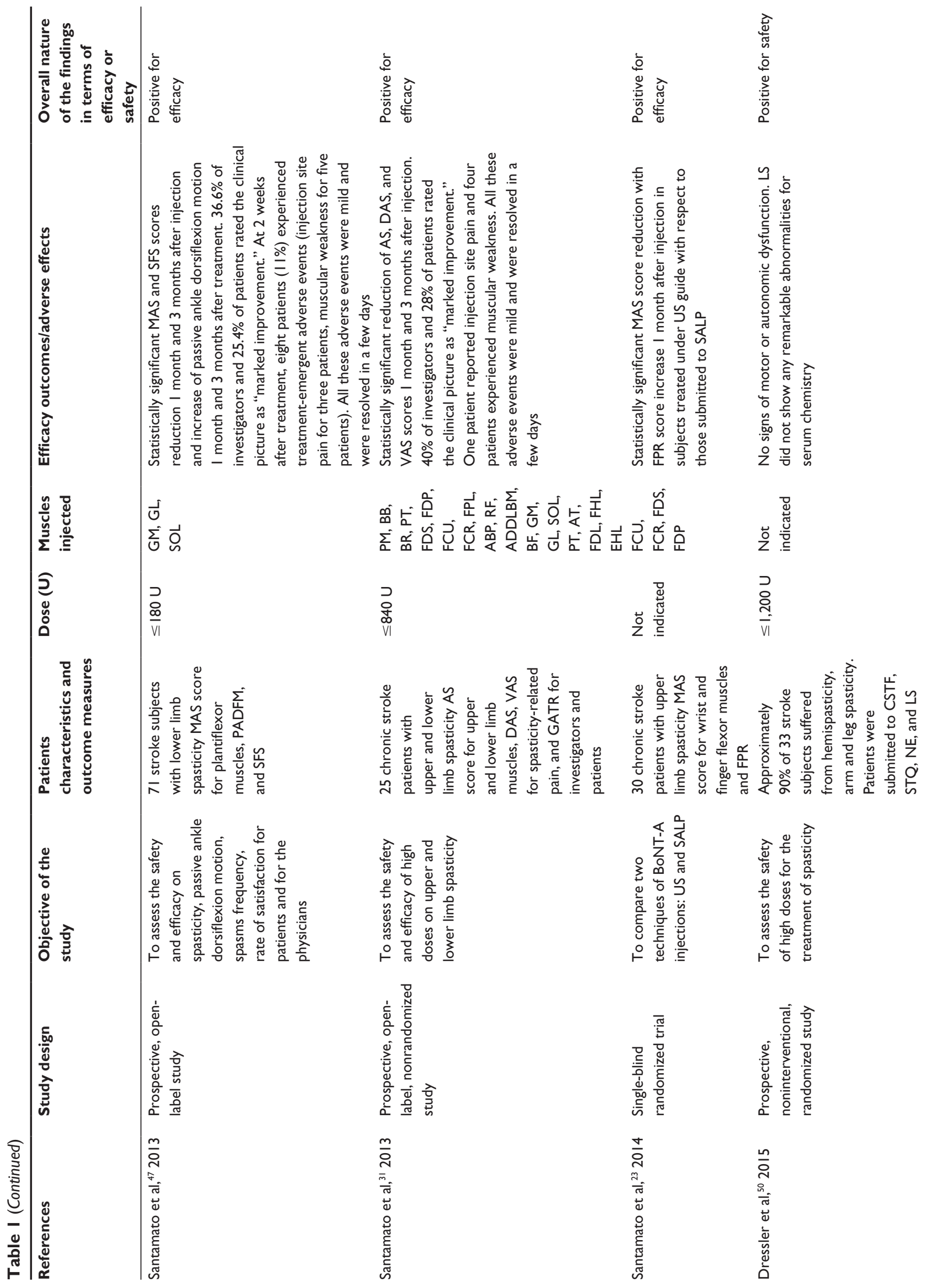




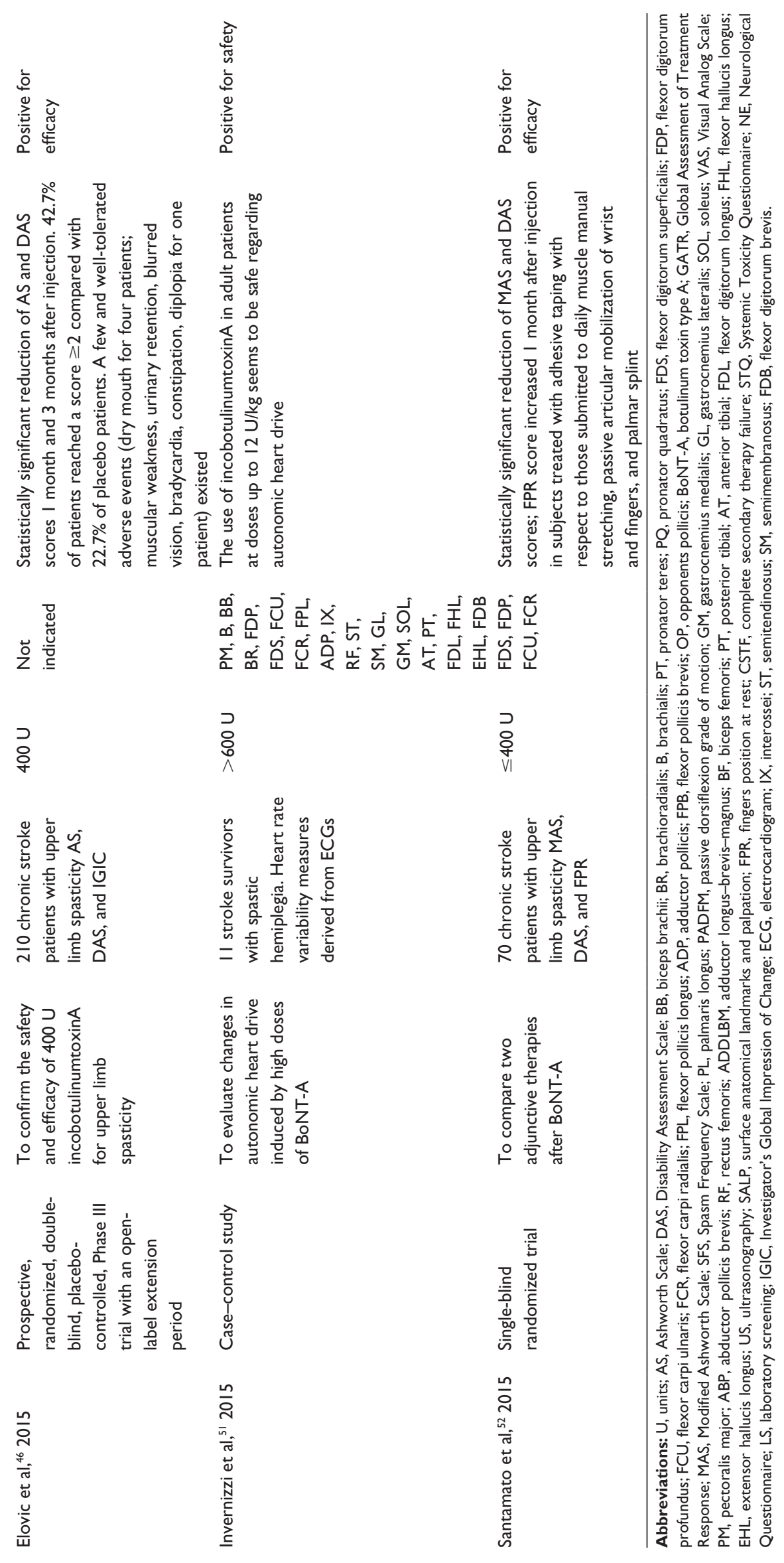


patients after each treatment cycle with few treatment-related adverse events ( $11 \%$ of patients). A prospective, randomized, observer-blind trial evaluated the efficacy and safety of two dilutions of incobotulinumtoxin $\mathrm{A}(50 \mathrm{U} / \mathrm{mL}$ or $20 \mathrm{U} / \mathrm{mL})$ in 192 patients with upper limb spasticity of diverse etiology. ${ }^{45}$ Of these, 168 subjects were stroke survivors and were randomized to either $50 \mathrm{U} / \mathrm{mL}$ or $20 \mathrm{U} / \mathrm{mL}$ of BoNT-A dilutions and treated with a maximum total dose of $495 \mathrm{U}$. Changes in functional disability and muscle tone from baseline to week 4 after incobotulinumtoxiA treatment have been assessed: a $\geq 1$-point reduction was observed on the DAS score in $57.1 \%$ and on the AS score in $>62.2 \%$ of patients. No differences in clinical effects were reported between subjects treated with $20 \mathrm{U} / \mathrm{mL}$ or $50 \mathrm{U} / \mathrm{mL}$ dilution. Both the patients $(80.2 \%)$ and the investigators $(89.0 \%)$ improved the clinical condition from "mildly" to "very markedly."

Elovic et al confirmed the efficacy and safety of incobotulinumtoxinA for poststroke upper limb spasticity in another 12-week main period of a prospective, randomized, doubleblind, placebo-controlled, Phase III trial with an open-label extension period. ${ }^{46}$ In the first phase of this trial, stroke survivors with a typical clinical pattern of flexed elbow, flexed wrist, and clenched fist with muscle tone $\geq 2$ on the AS at each site were treated with a single injection of $400 \mathrm{U}$ total dose of incobotulinumtoxinA $(n=210)$ or placebo $(n=107)$ for the safety evaluation set. Of these, 259 subjects were randomized after the amended protocol came into effect and were included in the full analysis set (incobotulinumtoxinA, $\mathrm{n}=171$; placebo, $\mathrm{n}=88$ ). Spasticity reduction measured with AS was the primary outcome measure, whereas the Investigator's Global Impression of Change (IGIC) using a 7-point balanced Likert scale was a coprimary outcome. The DAS was a secondary outcome variable. Patients reported a statistically significant reduction in hypertone and disability considering $\geq 1$-point improvement in the AS and DAS scores from baseline. In fact, after 1 month, the subjects submitted to incobotulinumtoxinA therapy showed a greater spasticity $(P<0.001)$ and disability $(P=0.007)$ reduction than placebo. IGIC confirmed the superiority of incobotulinumtoxinA versus placebo $(P=0.003)$. Adverse effects developed both in the BoNT-A group (22.4\%) and in the placebo group (16.8\%) were similar to other previous studies and were considered to be related to treatment, but there were no study discontinuations due to adverse events. Subjects enrolled in the trial continued into a 36-week, open-label extension period to receive three further treatments with $400 \mathrm{U}$ of incobotulinumtoxinA at fixed 12-week intervals, but to date no data are published.
The effectiveness of incobotulinumtoxinA to reduce spasticity of ankle plantar flexor muscles has been reported in a prospective open-label study conducted among 71 stroke survivors treated with neurotoxin administrated into soleus, medial, and lateral gastrocnemius muscles with a maximum total dose of $180 \mathrm{U}$ (dose range 25-100 U for each muscle). Patients reported a statistically significant reduction in muscle tone and spasms daily increasing passive ankle dorsiflexion movement at 30 days, persisting also at 90 days of follow-up. Two weeks after treatment, adverse events were reported by eight patients (11\%) (injection site pain and muscular weakness), which were all mild in intensity and resolved shortly. ${ }^{47}$ Another open-label study explored the safety and the efficacy of repeated incobotulinumtoxinA injections for 1 year while evaluating the functional disability and quality of life in 20 subjects with upper limb spasticity after stroke. Adverse events were assessed, and efficacy was measured by Modified Ashworth Scale (MAS) for spasticity, Spasm Frequency Scale (SFS) for the daily spasms, and DAS score. Every 3 months, the patients were injected into their upper limb muscles. During the reinjection time, the total dose was reduced with respect to the first injections, ranging from 160-450 U to 120-350 U, in parallel to the improvement of clinical picture. One year after treatment with the initial incobotulinumtoxinA, muscle tone, determined using the MAS, was significantly reduced in all the treated muscle groups $(P<0.001)$. In addition, DAS and SFS scores were also significantly reduced $(P<0.001$ for all). After the first and the last treatment, no differences regarding the effectiveness of MAS, DAS, and SFS reduction have been found, confirming the persistence of the incobotulinumtoxinA action during long-term therapy. Moreover, the authors reported no adverse events in these patients. ${ }^{48}$

A recent interest about the poststroke spasticity is the employment of BoNT-A in the early phase of stroke to reduce the disabling muscle contracture and stiffness in the paretic arm. Hesse et al conducted a randomized, single-blind, pilot study in 18 patients with increased finger flexors tone between 4 weeks and 6 weeks after a stroke. ${ }^{49}$ Participants were divided into group A and group B: group A received $150 \mathrm{U}$ of incobotulinumtoxinA (100 U into deep and superficial finger flexors; $50 \mathrm{U}$ into flexor carpi radialis and ulnaris muscles) under ultrasound-guided plus rehabilitation therapy for 4 weeks and group B received 4 weeks of a multiprofessional rehabilitation program without BoNT-A treatment. One month after treatment, the muscle tone of the finger flexors measured with MAS was significantly lower in patients who received incobotulinumtoxinA than in 
patients who had not received any injections $(P=0.001)$. The effects lasted for 6 months $(P=0.025)$. Moreover, 1 month after the BoNT-A administration, the whole arm muscle tone measured with REPAS (Resistance to Passive movement summary rating scale) was significantly lower in the group A $(P=0.003)$, whereas it missed the chosen level of significance after 6 months $(P=0.013)$. The disability sum score was significantly lower after 1 month $(P=0.023)$ and 6 months $(P=0.013)$. The Fugl-Meyer scores did not show any differences between groups after 1 month $(P=0.788)$ and 6 months $(P=0.857)$. The authors concluded that early treatment with incobotulinumtoxinA could potentially prevent the development of contractures reducing muscle stiffness in the longer term and that further placebo-controlled studies are needed.

The potentially reduced formation of antibodies development due to the absence of complexing proteins was the reason to perform several trials on the use of high doses of this pure neurotoxin although the European incobotulinumtoxinA product label recommends a maximum dose of $400 \mathrm{U}$ for the treatment of upper limb poststroke spasticity. A prospective, nonrandomized, open-label study described the safety and efficacy of higher doses (ranging from $750 \mathrm{U}$ to $840 \mathrm{U}$ ) of incobotulinumtoxinA in 25 consecutive subjects with upper and lower limb poststroke spasticity. ${ }^{31}$ The patients were treated under ultrasound guide in several muscles of the upper and lower limbs, reporting after 30 days of follow-up, a significant reduction in disability, spasticity-related pain, and muscle tone measured with DAS, Visual Analog Scale, and AS, respectively $(P=0.0000)$. At 90 days of follow-up, the incobotulinumtoxinA treatment effects were still significant $(P=0.0000)$. Only $16 \%$ of patients experienced treatmentemergent adverse events (injection site pain and muscular weakness). All these adverse events were mild and resolved in a few days. In a prospective, open-label noninterventional study, Dressler et al evaluated safety outcomes in a randomly selected population of 54 patients suffering from spasticity of several etiologies (15 suffered with hemispasticity, 13 with arm spasticity, 12 with tetraspasticity, 9 with paraspasticity, and 5 with leg spasticity): $\sim 90 \%$ of subjects with hemispasticity and arm and leg spasticity were stroke survivors. The patients were submitted to the "high-dose" group, receiving a single mean dose of 570.1 U (range 400-1,200 U) of incobotulinumtoxinA and the "regular dose" group with a single mean dose of 153.2 U (range 60-200 U) of the same neurotoxinA. No signs of motor or autonomic dysfunction that were attributable to incobotulinumtoxinA treatment have been reported with the use of the systemic toxicity patient questionnaire and with neurological examination. No patient developed production of antibodies and secondary treatment failure. $^{50}$

The safety of high doses of incobotulinumtoxinA was assessed also in a recent study evaluating possible changes in autonomic heart drive induced by $>600 \mathrm{U}$ of incobotulinumtoxinA injected into eleven stroke survivors with spastic hemiplegia. An electrocardiographic examination performed 24 hours before and 10 days after the injections did not show relevant effects on autonomic drive directed to the heart, demonstrating an absence of systemic diffusion also at high doses. ${ }^{51}$ Injection technique represents an important guide to maximize the precision in target muscles, avoiding the spread of neurotoxin in other sites. In the last few years, several studies have been published about the use of ultrasound guide during the BoNT treatment. A correct use of ultrasound guide permits a good result for the outcome measures employed to value disability of the patient injected. In fact, a comparison study between ultrasound guide (group A patients) and manual needle placement identifying target muscles by surface anatomical landmarks and palpation (group B patients) has been conducted on 30 stroke survivors with upper limb spasticity injected with incobotulinumtoxinA. ${ }^{23}$ Statistically significant improvement in patients' clinical picture has been found more in group A than in group B. One month after the incobotulinumtoxinA injection, MAS score for wrist flexor muscles was reduced $(P=0.0211)$ as well as MAS score for finger flexor superficialis muscles $(P=0.0124)$ and for finger flexor profundus muscles $(P=0.0124)$ with an improvement of finger position at rest $(P=0.0041)$, confirming the important role of a guide during the injections and the efficacy of incobotulinumtoxinA to reduce chronic spasticity. Finally, the effectiveness of incobotulinumtoxinA has been evaluated in 70 patients with wrist and finger flexor muscles spasticity treated with BoNT-A and randomly submitted to adhesive taping (group A) or daily muscle manual stretching, passive articular mobilization of wrist and fingers, and palmar splint (group B) for 10 days. MAS, DAS, and fingers position at rest were measured at baseline, after 2 weeks and after 1 month from the treatment session. After 2 weeks, subjects in group A reported a significantly greater decrease in spasticity scores (MAS fingers: $P<0.0001$; MAS wrist: $P<0.01)$ with an improvement in fingers position at rest $(P<0.001)$ compared with group B patients; these results persisted also 1 month after in spasticity, disability scores (MAS fingers: $P<0.001$; MAS wrist: $P<0.01$; DAS: $P<0.01)$ and fingers position at rest $(P<0.0001)$ with respect to Group B. ${ }^{52}$ 


\section{Discussion}

The cumulative body of evidence coming from the 12 studies selected in the present review article suggested that incobotulinumtoxinA, as well as onabotulinumtoxin $\mathrm{A}$ and abobotulinumtoxinA, appeared to be safe and efficacious in reducing focal spasticity after stroke. In fact, several studies and meta-analyses demonstrated that BoNT-A injections represent the first choice for the treatment of focal spasticity. ${ }^{26,27}$ A recent European consensus established that a dose of $\sim 600 \mathrm{U}$ of onabotulinumtoxinA/incobotulinumtoxinA or up to $1,500 \mathrm{U}$ of abobotulinumtoxin $\mathrm{A}$ may be safe and well tolerated in poststroke spasticity, ${ }^{28}$ even if higher doses of BoNT-A have been used, especially in the case of severe spasticity in the upper and lower limbs..$^{31,32,53}$

Although controversy exists about the improvement in motor function relative to spasticity reduction after BoNT-A treatment, the rate of satisfaction for clinicians, patients, and caregivers is very high. In fact, it is important, in the case of spasticity, to consider not only the motor impairment and loss of dexterity but also the associated clinical phenomena, for example, pain, disability, and discomfort sensations that can be reduced with BoNT-A injection. For this reason, the effect of BoNT-A therapy must be evaluated globally, as confirmed by the patient's considerations about the clinical results after the injections. Many times, the subjects treated with BoNT-A claim to have had a positive effect, also when there was not a great improvement in the reduction of motricity or spasticity. Probably, this subjective sensation is related to the reduction of pain, rigidity, heaviness of spastic limb, and in these cases, several outcome measures other than MAS or AS should be used to evaluate patient' sensations, quality of life, or potential benefit for caregivers after BoNT-A injection.

In the last few years, there has been a major interest in this topic; hence, several studies demonstrated the effectiveness and safety of incobotulinumtoxinA for spasticity after stroke reduction, including the rate of satisfaction of patients treated, clinicians, and caregivers. Other outcome variables included the global assessment of efficacy using some tasks of the Caregiver Burden Scale ${ }^{43}$ or a 7-point balanced Likert scale (IGIC) ${ }^{46}$ and the efficacy of the treatment for investigators and patients using a 9-point scale. ${ }^{31}$ In all studies reviewed in the present article, there was a positive result about these outcomes. This was in line with other studies about the use of BoNT-A therapy in stroke survivors, in which the improvements in quality of life, caregiver burden, and clinicians' satisfaction are key measures of success during the follow-up. For example, the possibility of using Short Form 36 questionnaire before and after injection consents to obtain the real consideration of the patients with spasticity on specific items, including physical functioning, role limitations due to physical health and emotional problems, bodily pain, general health perceptions, vitality, social functioning, and mental health according to the World Health Organization and International Classification of Functioning, Disability and Health. In a recent prospective, observational, and interventional 12-weeks trial on 27 stroke patients submitted to BoNT-A injections, a significant improvement in patients' perceived health-related quality of life was found in addition to objectively and subjectively measured motor functions. ${ }^{54}$ On the contrary, the Short Form 36 questionnaire did not modify after BoNT-A injection in another study, probably for a lack of improvement in the activity domain, and consequently, this can appear as a failure for patients' expectations..$^{55}$ The quality of life also did not change in 96 stroke patients submitted to abobotulinumtoxinA therapy or placebo, whereas a significantly greater reduction in spasticity, which translated into higher Global Attainment Scaling scores and greater global benefit, was obtained in another study. ${ }^{56}$ The results from two cross-sectional surveys conducted on 79 patients with poststroke spasticity treated with BoNT-A and 105 physicians, recently published, confirmed their satisfaction for the treatment: overall, $40.5 \%$ of patients were very satisfied, $48.1 \%$ were somewhat satisfied, and only $11.4 \%$ were not at all satisfied with BoNT treatment, whereas most of the 105 participating physicians' were moderately $(57.7 \%)$ or very $(36.5 \%)$ satisfied with BoNT treatment. ${ }^{57}$ Thus, a specific goal should be set for each patient before BoNT-A injection to demonstrate the effectiveness of the treatment not only for spasticity reduction but also for other domains. With regard to the safety of incobotulinumtoxinA, doses from $<600 \mathrm{U}$ up to $840 \mathrm{U}$ have been considered safe and well tolerated for the all patients injected in all reviewed studies 2 weeks or 4 weeks after the treatment. All adverse effects related to the treatment were mild (ie, injection site pain and muscular weakness) and were resolved in a few days. To date, it is not possible to affirm with certainty that the absence of complexing proteins of incobotulinumtoxinA can reduce the risk of the development of antibodies to neurotoxin protein interfering with pharmacological activity and reducing the therapeutic effect partially or completely. Several further studies are needed on larger sample of patients submitted to frequent injections or higher doses with longer follow-up to draw definitive conclusions.

Targeting the right site for BoNT-A injections is important to increase the effect. To date, there are no recommendations about which techniques are most suitable during the treatment; however, ultrasound guide permits in real time 
the evaluation of muscle characteristics identifying fat and fibrosis area. This issue is very notable because structural and functional changes can occur in skeletal muscle secondary to spasticity and in response to altered use and neural activation patterns after stroke. ${ }^{58,59}$ These pathological changes are more evident in older age. In fact, sarcopenia, loss of muscle mass, muscle cellular atrophy due to fiber size reduction, fat distribution, and fibrosis development are also amplified with aging and stroke-related inactivity, reducing the number of motor endplates and representing the reason for botulinum toxin therapy failure. These biological processes produce a muscle stiffness increase and could provide an explanation for an "increased resistance to stretch" during spasticity evaluation not necessarily related to upper motor neuron syndrome. Therefore, in this case, the effect of BoNT-A is very much reduced, and it may further be responsible for muscle changes due to inactive muscle fibers from the nerve block. In fact, previous studies showed that skeletal muscle properties did not recover after BoNT-A injection, causing muscle mass and strength loss and reduction of percentage of contractile material. ${ }^{60,61}$ These potentially harmful effects should be considered by clinicians before BoNT-A administration into spastic muscles, although it has been shown that an exercise protocol after BoNT-A treatment can help alleviate muscle atrophy and weakness.

\section{Conclusion}

The studies about the use of incobotulinumtoxinA in patients with upper and lower limb spasticity after stroke confirmed previous findings on the efficacy and safety of BoNT-A injections for spasticity treatment, reducing functional disability and improving quality of life. The effectiveness to reduce hypertone is largely demonstrated, but it is necessary to choose an individual primary functional target before starting with BoNT-A treatment, considering that spasticity reduction is not related to improvement of motor function in upper or lower limbs and that the nerve block is highly recommended in the absence of skeletal muscle changes secondary to spasticity and in response to altered use and neural activation patterns after stroke. The possibility of using a highly purified incobotulinumtoxinA potentially free from complexing proteins and, thus, associated with a relatively low risk of immunogenicity may represent a therapeutic advantage for a long-term treatment and for the use of high doses. Finally, a specialized training on patient's assessment, BoNT-A dosage, injection technique, and side effects knowledge before taking care of patients with spasticity after stroke should be required to confirm the effectiveness of this treatment.

\section{Disclosure}

The author reports no conflicts of interest. The author alone is responsible for the content and writing of the paper.

\section{References}

1. Wallesch C-W, Maes E, Lecomte P, Bartels C. Feasibility study on pharmacoeconomics of botulinum toxin A (Botox) in spasticity following stroke. 3rd Eur Botulinum Toxin Symp Abstr. 1997;1:4.

2. Watkins CL, Leathley MJ, Gregson JM, Moore AP, Smith TL, Sharma AK. Prevalence of spasticity post stroke. Clin Rehabil. 2002; 16:515-522.

3. Lundström E, Terént A, Borg J. Prevalence of disabling spasticity 1 year after first-ever stroke. Eur J Neurol. 2008;15(6):533-539.

4. Sommerfeld DK, Eek EU, Svensson AK, Widen Holmqvist L, von Arbin MH. Spasticity after stroke: its occurrence and association with motor impairments and activity limitations. Stroke. 2004;35(1): 134-139.

5. Welmer AK, von Arbin MH, Widen Holmqvist L, Sommerfeld DK. Spasticity and its association with functioning and health-related quality of life 18 months after stroke. Cerebrovasc Dis. 2006;21: 247-253.

6. Tenniglo MJ, Nederhand MJ, Prinsen EC, Nene AV, Rietman JS, Buurke JH. Effect of chemodenervation of the rectus femoris muscle in adults with a stiff knee gait due to spastic paresis: a systematic review with a meta-analysis in patients with stroke. Arch Phys Med Rehabil. 2014;95(3):576-587.

7. Ward AB. Spasticity treatment with botulinum toxins. J Neural Transm. 2008;115(4):607-616.

8. Smania N, Picelli A, Munari D, et al. Rehabilitation procedures in the management of spasticity. Eur J Phys Rehabil Med. 2010;46(3): 423-438.

9. Hulme A, MacLennan WJ, Ritchie RT, John VA, Shotton PA. Baclofen in the elderly stroke patient its side-effects and pharmacokinetics. Eur J Clin Pharmacol. 1985;29(4):467-469.

10. Dvorak EM, Ketchum NC, McGuire JR. The underutilization of intrathecal baclofen in poststroke spasticity. Top Stroke Rehabil. 2011;18(3): 195-202.

11. Dahm LS, Beric A, Dimitrijevic MR, Wall PD. Direct spinal effect of a benzodiazepine (midazolam) on spasticity in man. Stereotact Funct Neurosurg. 1989;53(2):85-94.

12. Cutter NC, Scott DD, Johnson JC, Whiteneck G. Gabapentin effect on spasticity in multiple sclerosis: a placebo-controlled, randomized trial. Arch Phys Med Rehabil. 2000;81(2):164-169.

13. Malanga G, Reiter RD, Garay E. Update on tizanidine for muscle spasticity and emerging indications. Expert Opin Pharmacother. 2008; 9(12):2209-2215.

14. Joynt RL. Dantrolene sodium: long-term effects in patients with muscle spasticity. Arch Phys Med Rehabil. 1976;57(5):212-217.

15. Lakhan SE, Rowland M. Whole plant cannabis extracts in the treatment of spasticity in multiple sclerosis: a systematic review. BMC Neurol. 2009;9:59.

16. Malhotra S, Rosewilliam S, Hermens H, Roffe C, Jones P, Pandyan AD. A randomized controlled trial of surface neuromuscular electrical stimulation applied early after acute stroke: effects on wrist pain, spasticity and contractures. Clin Rehabil. 2013;27(7):579-590.

17. Bovend'Eerdt TJ, Newman M, Barker K, Dawes H, Minelli C, Wade DT. The effects of stretching in spasticity: a systematic review. Arch Phys Med Rehabil. 2008;89(7):1395-1406.

18. Mazarakis NK, Ughratdar I, Vloeberghs MH. Excellent functional outcome following selective dorsal rhizotomy in a child with spasticity secondary to transverse myelitis. Childs Nerv Syst. 2015;31(11): 2189-2191.

19. Eppinger MA, Berman CM, Mazzola CA. Selective dorsal rhizotomy for spastic diplegia secondary to stroke in an adult patient. Surg Neurol Int. 2015;6:111. 
20. Carda S, Bertoni M, Zerbinati P, Rossini M, Magoni L, Molteni F. Gait changes after tendon functional surgery for equinovarus foot in patients with stroke: assessment of temporo-spatial, kinetic, and kinematic parameters in 177 patients. Am J Phys Med Rehabil. 2009;88(4):292-301.

21. Gracies JM, Elovic E, McGuire J, Simpson DM. Traditional pharmacological treatments for spasticity. Part I: local treatments. Muscle Nerve Suppl. 1997;6:S61-S91.

22. Dolly JO, Aoki KR. The structure and mode of action of different botulinum toxins. Eur J Neurol. 2006;13(suppl 4):1-9.

23. Santamato A, Micello MF, Panza F, et al. Can botulinum toxin type A injection technique influence the clinical outcome of patients with poststroke upper limb spasticity? A randomized controlled trial comparing manual needle placement and ultrasound-guided injection techniques. J Neurol Sci. 2014;347(1-2):39-43.

24. Franceschini M, Iocco M, Molteni F, Santamato A, Smania N; Italian Spasticity Study Group. Management of stroke patients submitted to botulinum toxin type A therapy: a Delphi survey of an Italian expert panel of specialist injectors. Eur J Phys Rehabil Med. 2014;50(5): $525-533$.

25. Das TK, Park DM. Effect of treatment with botulinum toxin on spasticity. Postgrad Med J. 1989;65:208-210.

26. Brashear A, Gordon MF, Elovic E, et al. Botox Post-Stroke Spasticity Study Group. Intramuscular injection of botulinum toxin for the treatment of wrist and finger spasticity after a stroke. N Engl J Med. 2002; 347:395-400.

27. Simpson DM, Alexander DN, O'Brien CF, et al. Botulinum toxin type A in the treatment of upper extremity spasticity: a randomized, doubleblind, placebo-controlled trial. Neurology. 1996;46(5):1306-1310.

28. Wissel J, Ward AB, Erztgaard P, et al. European consensus table on the use of botulinum toxin type A in adult spasticity. J Rehabil Med. 2009;41(1):13-25.

29. Sheean G, Lannin NA, Turner-Stokes L, Rawicki B, Snow BJ. Botulinum toxin assessment, intervention and after-care for upper limb hypertonicity in adults: international consensus statement. Eur J Neurol. 2010; 17(suppl 2):74-93.

30. Simpson DM, Gracies JM, Graham HK, et al. Assessment: Botulinum neurotoxin for the treatment of spasticity (an evidence-based review): report of the Therapeutics and Technology Assessment Subcommittee of the American Academy of Neurology. Neurology. 2008;70(19):1691-1698.

31. Santamato A, Panza F, Ranieri M, et al. Efficacy and safety of higher doses of botulinum toxin type A NT 201 free from complexing proteins in the upper and lower limb spasticity after stroke. J Neural Transm. 2013;120(3):469-476.

32. Santamato A, Micello MF, Ranieri M, et al. Employment of higher doses of botulinum toxin type A to reduce spasticity after stroke. $J$ Neurol Sci. 2015;350(1-2):1-6.

33. Albanese A. Terminology for preparations of botulinum neurotoxins: what a difference a name makes. JAMA. 2011;305:89-90.

34. Dressler D, Mander GJ, Fink K. Equivalent potency of Xeomin ${ }^{\circledR}$ and Botox ${ }^{\circledR}$. Mov Disord. 2008;1(23 suppl):S20-S21.

35. Dressler D, Mander G, Fink K. Measuring the potency labelling of onabotulinumtoxinA (Botox( $(\circledR))$ and incobotulinumtoxinA (Xeomin( $(\circledR))$ in an LD50 assay. J Neural Transm. 2012;119:13-15.

36. Odergren $\mathrm{T}$, Hjaltason $\mathrm{H}$, Kaakkola $\mathrm{S}$, et al. A double blind, randomised, parallel group study to investigate the dose equivalence of Dysport ${ }^{\circledR}$ and Botox ${ }^{\circledR}$ in the treatment of cervical dystonia. J Neurol Neurosurg Psychiatry. 1998;64:6-12.

37. Frevert J. Content of botulinum neurotoxin in Botox ${ }^{\circledR} / V^{\text {Vistabel }}{ }^{\circledR}$, Dysport $^{\circledR} /$ Azzalure $^{\circledR}$, and $\mathrm{Xeomin}^{\circledR} /$ Bocouture $^{\circledR}$. Drugs R D. 2010; 10(2):67-73.

38. Grein S, Mander GJ, Fink K. Stability of botulinum neurotoxin type A, devoid of complexing proteins. Botulinum J. 2011;2(1):49-57.

39. Eisele KH, Fink K, Vey M, Taylor HV. Studies on the dissociation of botulinum neurotoxin type A complexes. Toxicon. 2011;57(4):555-565.

40. Carli L, Montecucco C, Rossetto O. Assay of diffusion of different botulinum neurotoxin type a formulations injected in the mouse leg. Muscle Nerve. 2009;40(3):374-380.
41. Santamato A, Ranieri M, Panza F, et al. Effectiveness of switching therapy from complexing protein-containing botulinum toxin type A to a formulation with low immunogenicity in spasticity after stroke: a case report. J Rehabil Med. 2012;44(9):795-797.

42. Merz Pharmaceuticals GmbH [webpage on the Internet]. Xeomin ${ }^{\circledR}$ US Prescribing Information [updated July 2011]. Available from http:// www.accessdata.fda.gov/drugsatfda_docs/label/2013/125360s045lbl. pdf. Accessed October 13, 2014.

43. Kanovský P, Slawek J, Denes Z, et al. Efficacy and safety of botulinum neurotoxin NT 201 in poststroke upper limb spasticity. Clin Neuropharmacol. 2009;32(5):259-265.

44. Kaňovský P, Slawek J, Denes Z, et al. Efficacy and safety of treatment with incobotulinum toxin A (botulinum neurotoxin type A free from complexing proteins; NT 201) in post-stroke upper limb spasticity. $J$ Rehabil Med. 2011;43(6):486-492.

45. Barnes M, Schnitzler A, Medeiros L, Aguilar M, Lehnert-Batar A, Minnasch P. Efficacy and safety of NT 201 for upper limb spasticity of various etiologies - a randomized parallel-group study. Acta Neurol Scand. 2010;122(4):295-302.

46. Elovic EP, Munin MC, Kaňovský P, Hanschmann A, Hiersemenzel R, Marciniak C. Randomized, placebo-controlled trial of incobotulinumtoxinA for upper-limb post-stroke spasticity. Muscle Nerve. Epub 2015 Jul 22.

47. Santamato A, Micello MF, Panza F, et al. Safety and efficacy of incobotulinum toxin type A (NT 201-Xeomin) for the treatment of post-stroke lower limb spasticity: a prospective open-label study. Eur J Phys Rehabil Med. 2013;49(4):483-489.

48. Fiore P, Santamato A, Ranieri M, et al. Treatment of upper limb spasticity after stroke: one-year safety and efficacy of botulinum toxin type A NT201. Int J Immunopathol Pharmacol. 2012;25(1 suppl): $57 \mathrm{~S}-62 \mathrm{~S}$.

49. Hesse S, Mach H, Frohlich S, Behrend S, Werner C, Melzer I. An early botulinum toxin A treatment in subacute stroke patients may prevent a disabling finger flexor stiffness six months later: a randomized controlled trial. Clin Rehabil. 2012;26(3):237-245.

50. Dressler D, Saberi FA, Kollewe K, Schrader C. Safety aspects of incobotulinumtoxinA high-dose therapy. J Neural Transm. 2015;122(2): 327-333.

51. Invernizzi M, Carda S, Molinari C, Stagno D, Cisari C, Baricich A. Heart rate variability (HRV) modifications in adult hemiplegic patients after botulinum toxin type A (nt-201) injection. Eur J Phys Rehabil Med. 2015;51(4):353-359.

52. Santamato A, Micello MF, Panza F, et al. Adhesive taping vs daily manual muscle stretching and splinting after botulinum toxin type A injection for wrist and fingers spastic overactivity in stroke patients: a randomized controlled trial. Clin Rehabil. 2015;29(1):50-58.

53. Baricich A, Grana E, Carda S, Santamato A, Cisari C, Invernizzi M. High doses of onabotulinumtoxinA in post-stroke spasticity: a retrospective analysis. J Neural Transm. 2015;122(9):1283-1287.

54. Bergfeldt U, Sköld C, Julin P. Short Form 36 assessed healthrelated quality of life after focal spasticity therapy. J Rehabil Med. 2009;41(4):279-281.

55. Caty GD, Detrembleur C, Bleyenheuft C, Deltombe T, Lejeune TM. Effect of upper limb botulinum toxin injections on impairment, activity, participation, and quality of life among stroke patients. Stroke. 2009;40(7):2589-2591.

56. McCrory P, Turner-Stokes L, Baguley IJ, et al. Botulinum toxin A for treatment of upper limb spasticity following stroke: a multi-centre randomized placebo-controlled study of the effects on quality of life and other person-centred outcomes. J Rehabil Med. 2009;41(7): $536-544$

57. Bensmail D, Hanschmann A, Wissel J. Satisfaction with botulinum toxin treatment in post-stroke spasticity: results from two crosssectional surveys (patients and physicians). J Med Econ. 2014;17(9): 618-625.

58. Lieber RL, Steinman S, Barash IA, Chambers H. Structural and functional changes in spastic skeletal muscle. Muscle Nerve. 2004;29(5): 615-627. 
59. Hafer-Macko CE, Ryan AS, Ivey FM, Macko RF. Skeletal muscle changes after hemiparetic stroke and potential beneficial effects of exercise intervention strategies. J Rehabil Res Dev. 2008;45(2): 261-272.

60. Fortuna R, Horisberger M, Vaz MA, Herzog W. Do skeletal muscle properties recover following repeat onabotulinum toxin A injections? J Biomech. 2013;46(14):2426-2433.
61. Fortuna R, Vaz MA, Sawatsky A, Hart DA, Herzog W. A clinically relevant BTX-A injection protocol leads to persistent weakness, contractile material loss, and an altered mRNA expression phenotype in rabbit quadriceps muscles. J Biomech. 2015;48(10):1700-1706.

\section{Publish your work in this journal}

Neuropsychiatric Disease and Treatment is an international, peerreviewed journal of clinical therapeutics and pharmacology focusing on concise rapid reporting of clinical or pre-clinical studies on a range of neuropsychiatric and neurological disorders. This journal is indexed on PubMed Central, the 'PsycINFO' database and CAS, and is the official journal of The International Neuropsychiatric Association (INA). The manuscript management system is completely online and includes a very quick and fair peer-review system, which is all easy to use. Visit http://www.dovepress.com/testimonials.php to read real quotes from published authors.

Submit your manuscript here: http://www.dovepress.com/neuropsychiatric-disease-and-treatment-journal 